TP Periodica Polytechnica

Transportation Engineering

42(1), pp. 73-76, 2014

DOI:10.3311/PPtr. 7068

http://www.pp.bme.hu/tr/article/view/7068

Creative Commons Attribution (i)

RESEARCH ARTICLE

\section{Analysis of Traffic Conflict Characteristic at Temporary Reversible Lane}

\author{
Yi Cao / Zhongyi Zuo / Huizhi Xu
}

RECeIVEd 9 October 2013; Accepted 18 December 2013

\section{Abstract}

The characteristics of traffic conflicts occurring at the time of direction changes were analyzed to better understand the problems of a temporary reversible lane. Using Huangpu Road as the investigation site, data of traffic conflicts before and after direction changes were investigated. The data on traffic conflict characteristics were compared and analyzed with mathematical statistics. Using the regression analysis method, a prediction model of the traffic conflicts was constructed. This paper also discussed the utilization ratio problem for this kind of lane. The research showed obvious and regular differences between before and after direction changing in a temporary reversible lane. There is an observable relationship between the traffic conflict, flow, and density. The problem of the lower lane utilization ratio of a temporary reversible lane usually exists.

\section{Keywords}

traffic flow theory ' reversible lane ' conflict characteristic . lane utilization ratio

\section{Yi Cao}

School of Transportation Engineering, Dalian Jiaotong University, Huanghe Rd. 794 Dalian, China

Post-doctoral Station of Communication and Transportation Engineering, Dalian Maritime University, Linghai Rd.1 Dalian, China e-mail: caoyi820619@aliyun.com

\section{Zhongyi Zuo}

School of Transportation Engineering, Dalian Jiaotong University, Huanghe Rd. 794 Dalian, China

e-mail: zuozy@djtu.edu.cn

Huizhi Xu

Traffic College, Northeast Forestry University, Hexing Rd. 26 Harbin, China e-mail: stedu@126.com

\section{Introduction}

With the constant expansion of urban land into the Chinese countryside, the city's main traffic channels increasingly suffer from traffic tide phenomenon. Peng Liu (2011) show that traffic tide phenomenons can lead to an insufficient supply of traffic facilities for heavy traffic directions, and even cause traffic jams. The phenomenons can also lead to idle traffic resources for light-traffic directions. Lili Li (2008) indicated that, in order to make full use of the existing road resources, as well as relieve the inhomogeneity of direction caused by the traffic tide phenomenon, the main traffic channels of each city would need to use temporary reversible lanes for traffic organization. Therefore, it is necessary to quantitatively analyze the traffic operation characteristics of temporary reversible lane, for the purpose of revealing the internal rule of this new system of traffic organization. This study aims to lay a theoretical foundation for the implementation and improvement of this traffic organization system.

To date, both domestic and foreign scholars have studied the reversible lane. Dey Soumya (2011) introduced the reversible lane implementation for a city corridor at traffic peak periods for the purpose of improving American traffic flow. They also studied the application of the reversible lane in temporary emergency evacuations, traffic maintenance, and other special events. Using the National Cooperative Highway Research Program (NCHRP), Wolshon Brian (2006) studied the application range of the reversible lane, with a focus on assuring the security of the reversible lane, the influence on the environment, and the design and implementation requirements. Sheu J.B. (2001) studied the lanes- transform behavior when a reversible lane has been implemented. They also constructed a random predicting model for lanes-transform behavior. Here in China, Ziyou Gao (2007) proposed a lane adjustment method for roads with bidirectional traffic imbalance. They constructed a discrete bi-level programming model for allocating lanes in an urban traffic network. Based on the investigation of the ShuXi Road in ChengDu, China, Jian Chen (2008) used a traffic simulation model to demonstrate the feasibility of 
a temporary reversible lane. Using an ordered sample class and nonparametric regression, Xianyan Gong (2006) took on the traffic tide phenomenon and proposed a switching algorithm for travelling direction.

Although several studies on this topic, in general, have been carried out both here and abroad, most of them focused their attention on reversible lanes with clear signal indication and complete traffic marking, rather than addressing the temporary reversible lane. Moreover, there remains an insufficient body of quantitative studies on traffic conflict characteristics before and after the direction change at temporary reversible lanes. Given this status in the literature, in this study the existing problem of this kind of traffic organization has been analyzed quantitatively, using the sufficient traffic investigation data on temporary reversible lanes. There are significant theoretical and practical applications to be found when examining the implementation and improvement of temporary reversible lanes.

\section{Traffic investigation and data acquisition}

\subsection{The scheme of traffic survey}

Huangpu Road (from Lingyuan Street to Gaoneng Street) in Dalian is selected as the traffic investigation segment. The fulllength of this segment is $1.4 \mathrm{~km}$, with 6 lanes going in two directions. This road is one of the most important traffic channels from Dalian to Lushun. The phenomenon of traffic tide on this segment is easy to detect during peak traffic periods. Currently, this segment uses temporary reversible lanes to organize traffic without signals or a marking system for a formal reversible lane.

This investigation was carried out over 3 days, from Tuesday to Thursday. According to our research, the transform moment of this lane occurred at 16:30. Thus the time segment for this traffic investigation was between 16:00 and 17:00.

This investigation was divided into two stages, external observation and internal arrangement. Traffic video recordings were used to conduct the external observation, and mathematical statistics on the traffic data were used to conduct the internal arrangement.

\subsection{The traffic organization mode}

Temporary reversible lanes are a kind of traffic organization, which temporarily transform a lane's traffic direction by installing segregations or using manual commands. The lanes are usually implemented in accordance with the level of the traffic tide phenomenon, and installed without the signal or marking system or other auxiliary facilities typical in a formal reversible lane. According to observations of Huangpu Road, the implementation steps for this kind of traffic organization mode include 3 links.

\section{(1) Empty the lane.}

Using a special operations vehicle, several segregations are installed at the starting point of the temporary reversible lane.
The traffic flow in the direction of the light traffic converges into (n-1) lanes from $n$ lanes. While coordinating the vehicle traffic along the light traffic direction, several segregations are installed at the edge of the reversible lane. Throughout this process no vehicle will continue travelling in the reversible lane unless the operations vehicle reaches the ending point.

\section{Direction change and traffic supervision.}

When the operation vehicle arrives at the reversible lane end point, the passage of the heavy traffic direction is then permitted. The traffic flow for the direction of the heavy traffic is redistributed into $(n+1)$ lanes from $n$ lanes. In the temporary reversible lane, it is required that the traffic is monitored and controlled by traffic policemen.

\section{Removing segregation installations and resuming original traffic.}

When the traffic tide phenomenon has been relieved, it is necessary to resume original traffic operations. In this scenario, the vehicular travelling direction is opposite to link 1 . The direction should follow the direction of the heavy traffic from the end point to the starting point of the temporary reversible lane.

\subsection{Traffic data acquisition}

According to the internal arrangement of the video data obtained during the external observation, several traffic data were acquired, including the flow, density, and quantity of the traffic conflict.

\section{The analysis of traffic conflict}

\subsection{The comparative analysis of traffic conflict}

When compared to the formal reversible lane, the temporary reversible lane has no explicit or perfect signal and marking system. Therefore, when the temporary reversible lane is in use, traffic conflicts may be more severe.

In this study, the data of the traffic conflicts 15 minutes before and after the direction change at the temporary reversible lane is analyzed. The time interval, as shown in Table 1 , is every 3 minutes. Data analysis shows that when the temporary reversible lane is in operation the quantity of traffic conflicts is double that of the quantity of conflicts that occur normally.

\subsection{The cause analysis of traffic conflict}

Yonggang Wang (2012) showed that road segment traffic conflicts are usually caused by driving behavior. According to Wang, these are some of the common adverse driving behaviors: pressing-line driving, coercive lane switching, and illegal parking. Their constituent relationship is listed in figure 1.

In evaluating the relationship between traffic conflicts and adverse driving behavior, the videos show that pressing-line driving is not the main cause of traffic conflicts. It should be noted though that this behavior does occur more often than the 
Tab. 1. The quantity of traffic conflict

\begin{tabular}{cccc}
\hline \multicolumn{2}{c}{ Before direction changing } & \multicolumn{2}{c}{ After direction changing } \\
\hline Time interval & $\begin{array}{c}\text { quantity of } \\
\text { conflict }\end{array}$ & Time interval & $\begin{array}{c}\text { quantity of } \\
\text { conflict }\end{array}$ \\
\hline $16: 17-16: 20$ & 5 & $16: 38-16: 41$ & 8 \\
$16: 20-16: 23$ & 5 & $16: 41-16: 44$ & 10 \\
$16: 23-16: 27$ & 4 & $16: 44-16: 47$ & 11 \\
$16: 27-16: 30$ & 3 & $16: 47-16: 50$ & 10 \\
$16: 30-16: 33$ & 5 & $16: 50-16: 53$ & 9 \\
\hline
\end{tabular}

Tab. 2. The volume, density and traffic conflicts of reversible lane

\begin{tabular}{cccc}
\hline $\begin{array}{c}\text { Time interval } \\
(\mathrm{min})\end{array}$ & $\begin{array}{c}\text { Volume } \\
(\mathrm{pcu} / \mathrm{h})\end{array}$ & $\begin{array}{c}\text { Density } \\
(\mathrm{pcu} / \mathrm{km})\end{array}$ & $\begin{array}{c}\text { Conflicts } \\
\text { (times) }\end{array}$ \\
\hline $16: 38-16: 41$ & 227 & 98 & 8 \\
16:41-16:44 & 243 & 115 & 10 \\
$16: 44-16: 47$ & 251 & 126 & 11 \\
$16: 47-16: 50$ & 259 & 144 & 13 \\
16:50-16:53 & 249 & 127 & 9 \\
\hline
\end{tabular}

others. The main cause of conflicts, according to the videos, were coercive lane switching. Under heavy traffic conditions, one coercive lane change may lead to several vehicles in conflict with each other. These conflicts were found to occur in both the road segment and, more severely, at the starting point of the reversible lane. This is because the vehicles, regardless of their traffic direction, are all mixed up at the starting points. However, the traffic flows in two directions at the end point of the reversible lane are both distributive driving. So at the end point, traffic conflicts are fewer and farther between.

\subsection{The prediction model of traffic conflict}

The videos showed that driving conditions are better when the traffic flow and density are lower. During such conditions drivers do not have a need to coercively switch lanes. Likewise, even if drivers undertake some adverse driving behaviors it is unlikely that it will lead to a conflict with any neighboring vehicles. However, when there are more cars on the road in a denser flow, the videos showed that drivers are more likely to pre-emptively switch lanes, thus causing more traffic conflicts. Thus we can conclude that traffic conflicts occurring in a temporary reversible lane should relate with the traffic flow and density. The data supporting this conclusion can be found in Table 2. Using multiple regressions in Matlab, figure 2 can be sketched. The prediction model of the traffic conflict can also be obtained from Eq.1.

$$
c=0.5881 Q-1.805 K+0.0062 K^{2}-7.7711
$$

Where, $c$ is the quantity of traffic conflict (times). $Q$ is traffic volume (pcu/h). K is traffic density $(\mathrm{pcu} / \mathrm{km})$.

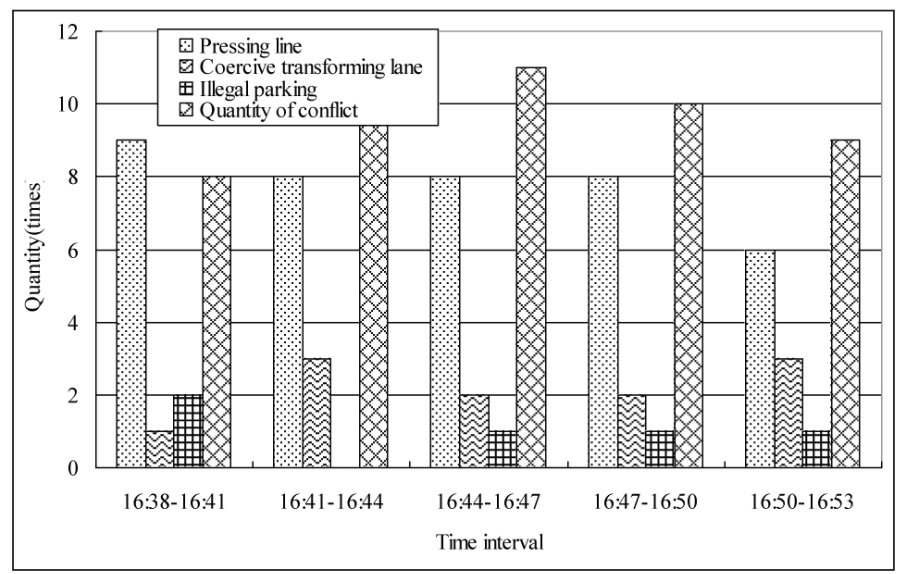

Fig. 1. The component of aggressive driving behavior at reversible lane

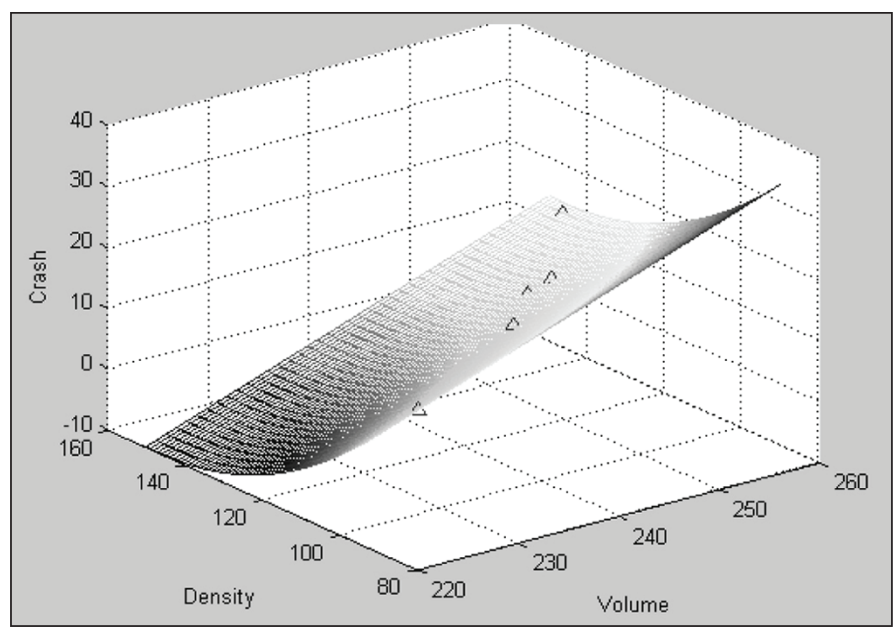

Fig. 2. The relationship between the traffic conflicts, flow and density

\section{The utilization characteristic}

In order to discuss the operating efficiency of the reversible lane, the concept of the lane utilization ratio is defined: the ratio in unit time of the vehicle quantity in the reversible lane to the vehicle quantity in its adjacent lane. This index represents the level of the reversible lane that can function as its adjacent lane. According to our video recordings, the utilization rate of the temporary reversible lane is significantly lower during the initial stage of direction changing, as shown in figure 3 and 4.

\section{The problems of the temporary reversible lane}

Temporary reversible lanes can, to some extent, adapt to the traffic tide phenomenon. The lanes also serve to temporarily relief traffic pressure, particularly when there is no adequate traffic management and control facilities. However, there are several problems with the lanes.

(1) The traffic management and control facilities for temporary reversible lanes are grossly inadequate. The traffic function of the lanes can be realized only partially with the installation of isolation facilities and traffic officers. 
2 The quantity of traffic conflicts at temporary reversible lanes is obviously more pronounced during the initial stage of direction changing. The main cause of these conflicts is adverse driving behavior generally and coercive lane switches in particular.

(3) The utilization rate of the temporary reversible lane is significantly lower during the initial stage of direction changing. There are only a few vehicles travelling in this lane.

\section{Conclusions}

The following conclusions are summarized based on the conducted analysis:

(1) The quantity of traffic conflicts in a temporary reversible lane is obviously greater than the conflicts that occur in an ordinary lane. There is a quantifiable relationship between traffic flow, density and the conflicts in a temporary reversible lane.

2 Temporary reversible lane present several problems: serious traffic conflicts, a low operating efficiency, and a low lane utilization rate. These problems can be relieved with a formal reversible lane.

3 Because of the traffic organization mode for the investigated segment, the acquired traffic data sustained the artificial influence of traffic policemen. This influence should be eliminated in future research.

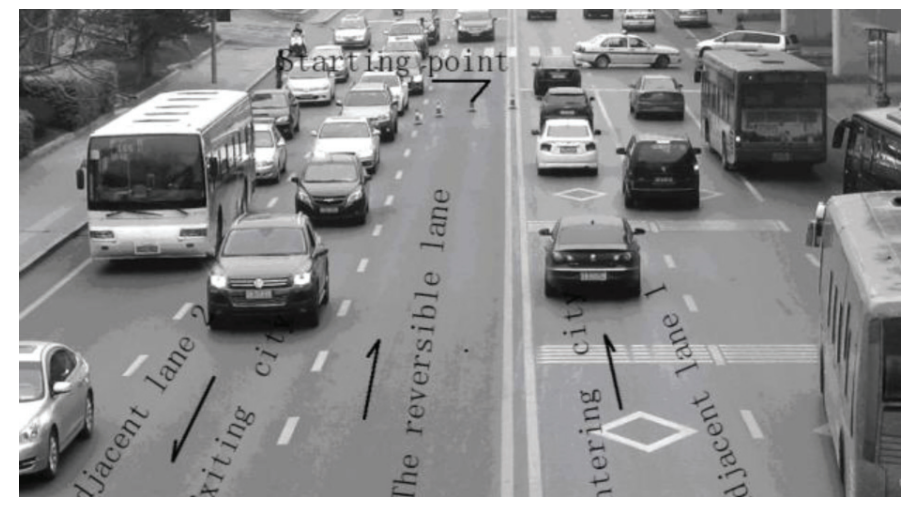

Fig. 3. The reversible lane with lower utilization ratio

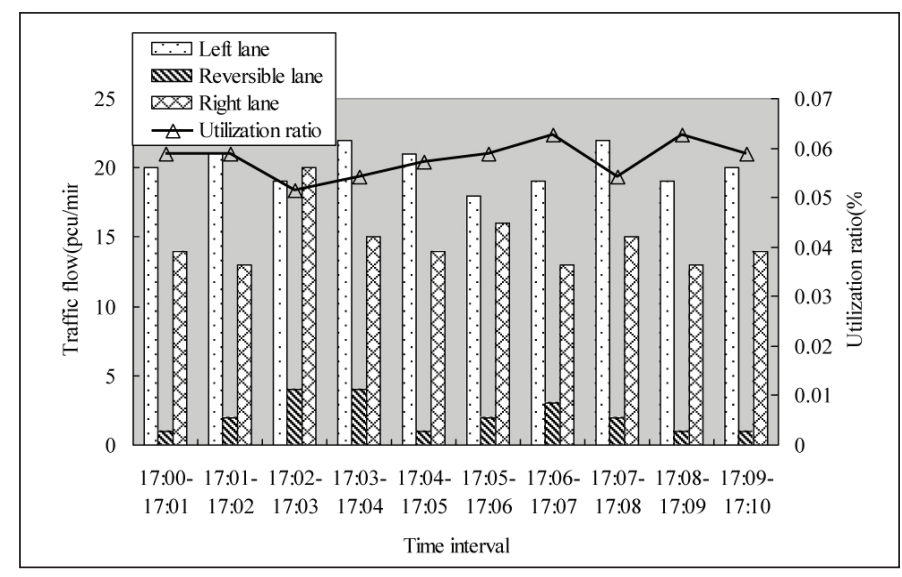

Fig. 4. Flow and utilization ratio of the reversible lane

\section{Acknowledgements}

This research was supported in part by the National Natural Science Foundation of China (51108137) and the Open Subject of Traffic Safety and Control Lab in Hebei Province (NO. JTKY2013002). We also acknowledge the editors and anonymous reviewers, for their detailed suggestions, precise comments and continuous helps.

\section{References}

1 Dey S., Mia J., Aden Y., Reversible lane operation for arterial roadways: the Washington, D.C, USA experience, Technical report, Institute of Transportation (ITE) Journal, Washington, D.C. 81 (5), (2011).

2 Zhang H., Gao Z., Optimization approach for traffic road network design problem. Chinese Journal of Management Science, 15 (2), 86-91 (2007).

3 Chen J., Huo Y., Study on setting design of variable lane on typical tide traffic road. Journal of Chong Qing Jiao Tong University, 27 (06), 1127-1130 (2008).

4 Li L., Qu Z., Wang D., Guidance method of the variable lane. Computer and Communications, 26 (5), 53-56 (2008).

5 Liu P., Liu Y., Study on characters of tidal transportation and relieve measures. Technology \& Economy in Areas of Communication, 13 (3), 92-94 (2011).

6 Sheu J. B., Ritchie S. G., Stochastic modeling and real-time prediction of vehicular lane-changing behavior. Transportation Research, 35 (7), 695-716 (2001). DOI: $\underline{10.1016 / \mathrm{S} 0191-2615(00) 00015-1}$
7 Brian W., Laurence L., Reversible lane systems: synthesis of practice. Journal of Transportation Engineering, 132 (12), 933-944 (2006). DOI: 10.1061/(ASCE)0733-947X(2006)132:12(933)

8 Gong X., Kang S., Study and application of traffic direction changing algorithm for urban tide traffic situation. Journal of Transportation System Engineering and Information Technology, 6 (6), 33-40 (2006).

9 Wang Y., Chen K., Liwei H., Killer tailgating: recommendation of traveling intervals between consecutive motor vehicles for rear end collision avoidance. The Arabian Journal for Science and Engineering, 37 (3), 619-630 (2012). DOI: $10.1007 / \mathrm{s} 13369-012-0200-\mathrm{y}$ 